\title{
Improving Locality in the Parallelization of Doacross Loops ${ }^{\star}$
}

\author{
María J. Martín ${ }^{1}$, David E. Singh ${ }^{2}$, Juan Touriño ${ }^{1}$, and Francisco F. Rivera ${ }^{2}$ \\ 1 Dep. of Electronics and Systems, University of A Coruña, Spain \\ \{mariam, juan\}@udc.es \\ 2 Dep. of Electronics and Computer Science, University of Santiago, Spain \\ \{david,fran\}@dec.usc.es
}

\begin{abstract}
In this work we propose a run-time approach for the efficient parallel execution of doacross loops with indirect array accesses by means of a graph partitioning strategy. Our approach focuses not only on extracting parallelism among iterations of the loop, but also on exploiting data access locality to improve memory hierarchy behavior and thus the overall program speedup. The effectiveness of our algorithm is assessed in an SGI Origin 2000.
\end{abstract}

\section{Introduction}

This work addresses the parallelization of doacross loops, that is, loops with loop-carried dependences. These loops can be partially parallelized by inserting synchronization primitives to force the memory access order imposed by these dependences. Unfortunately, it is not always possible to determine the dependences at compile-time as, in many cases, they involve input data that are only known at run-time and/or the access pattern is too complex to be analyzed.

There are in the literature a number of run-time approaches for the parallelization of doacross loops [1,2]34]. All of them follow an inspector-executor strategy and they differ on the kinds of dependences that are considered and the level of parallelism exploited (iteration-level or operation-level parallelism). A comparison between strategies based on iteration-level and operation-level parallelism is presented in [5]. The work shows experimentally that operation-level methods outperform iteration-level methods.

In this paper we present a new operation-level algorithm based on graph partitioning techniques. Our approach not only maximizes parallelism, but also (and basically) increases data locality to better exploit memory hierarchy in order to improve code performance. The target computer assumed throughout this paper is a CC-NUMA shared memory machine. We intend, on the one hand, to increase cache line reuse in each processor and, on the other hand, to reduce false sharing of cache lines, which is an important factor of performance degradation in CC-NUMA architectures.

\footnotetext{
* This work has been supported by the Ministry of Science and Technology of Spain and FEDER funds of the European Union (ref. TIC2001-3694-C02)
} 


\section{Run-Time Strategy}

Our method follows the inspector-executor strategy. During the inspector stage, memory access and data dependence information is collected. The access information, which determines the iteration partition approach, is stored in a graph structure. Dependence information is stored in a table called Ticket Table [1]. So, the inspector phase consists of three parts:

- Construction of a graph representing memory accesses. It is a non-directed weighted graph; both nodes and graph edges are weighted. Each node represents $m$ consecutive elements of array $A, m$ being the number of elements of $A$ that fit in a cache line. The weight of each node is the number of iterations that access that node for write. Moreover, a table which contains the indices of those iterations is assigned to each node. The edges join nodes that are accessed in the same iteration. The weight of each edge corresponds to the number of times that the pair of nodes is accessed in an iteration.

- Graph partitioning. The graph partitioning will result in a node distribution (and, therefore, an iteration distribution) among processors. Our aim is to partition the graph so that a good node balance is achieved and the number of edges being cut is minimum. Node balance results in load balance and cut minimization involves a decrease in the number of cache invalidations, as well as an increase in cache line reuse. Besides, as each node represents a cache line with consecutive elements of $A$, false sharing is eliminated. We have used the pmetis program [6] from the METIS software package to distribute the nodes among the processors according to the objectives described above.

- Creation of a Ticket Table containing data dependence information. The creation of the Ticket Table is independent of the graph construction and partitioning, and thus these stages can be performed in parallel.

The executor phase makes use of the dependence information recorded in the Ticket Table to execute, in each processor, the set of iterations assigned in the inspector stage. An array reference can be performed if and only if the preceding references are finished. All accesses to the target array are performed in parallel except for the dependences specified in the Ticket Table. The iterations with dependences can be partially overlapped because we consider dependences between accesses instead of between iterations. In [7] we propose an inspector that considers an iteration partitioning based on a block-cyclic distribution.

\section{Performance Evaluation}

In this section, the experimental results obtained for our strategy are evaluated and compared with the classical approach, an algorithm that uses a cyclic distribution of the iterations. The cyclic distribution maximizes load balancing and favors parallelism, without taking into account data access locality.

Although for illustrative purposes a loop with one read and one write per loop iteration will be used as case study, our method is a generic approach that can also be applied to loops with more than one indirect read access per iteration. 


\subsection{Experimental Conditions}

The parallel performance of the irregular doacross loop is mainly characterized by three parameters: loop size, workload cost and memory access pattern. In order to evaluate a set of cases as large as possible, we have used the loop pattern shown in Figure 1, where $N$ represents the problem size, the computational cost of the loop is simulated through the parameter $W$, and the access pattern is determined by the array $I N D E X$ and the size of array $A$. Examples of this loop pattern can be found in the solution of sparse linear systems (see, for instance, routines $l s o l, l d s o l$ and $l d s o l l$ of the Sparskit library [8]), where the loop size and the access pattern depend on the sparse coefficient matrix. These systems have to be solved in a wide variety of codes, including linear programming applications, process simulation, finite element and finite difference applications, and optimization problems, among others. Therefore, we have used in our experiments as indirection arrays the patterns of sparse matrices from the Harwell-Boeing collection [9] that appear in real codes. The test matrices are characterized in Figure 1 where the size of the indirection array $I N D E X$ is $2 \times N$, and $M$ is the size of array $A$.

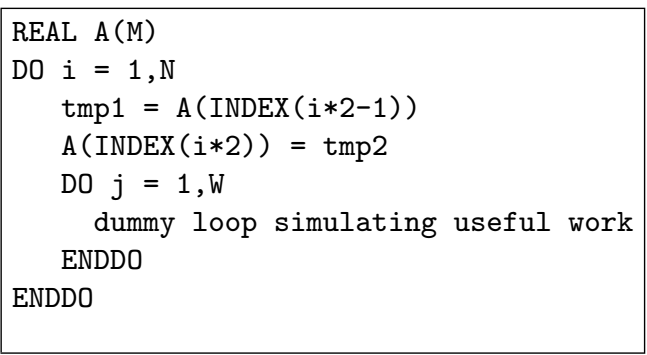

\begin{tabular}{|c|c|c|}
\hline & $N$ & $M$ \\
\hline \hline gemat1 & 23684 & 4929 \\
gemat12 & 16555 & 4929 \\
mbeacxc & 24960 & 496 \\
beaflw & 26701 & 507 \\
psmigr_2 & 270011 & 3140 \\
\hline
\end{tabular}

Fig. 1. Loop used as experimental workload and benchmark matrices

Our target machine is an SGI Origin 2000 CC-NUMA multiprocessor with R10k at $250 \mathrm{MHz}$. The R10k utilizes a two level cache hierarchy: L1 instruction and data caches of $32 \mathrm{~KB}$ each, and a unified L2 cache of 4MB (cache line size of 128 bytes). All tests were written in Fortran using OpenMP directives. All data structures were cache aligned. In our experiments, the cost per iteration of the outer loop of Figure 1 can be modeled as $\mathrm{T}(W)=8.02 \times 10^{-5}+8 \times 10^{-5} W$ ms. The cost per iteration depends on the application. For illustrative purposes, typical values of $W$ range from 5 to 30 using HB matrices for the loop patterns of the aforementioned Sparskit routines that solve sparse linear systems.

\subsection{Experimental Results}

We have used the R10k event counters to measure L1 and L2 cache misses as well as the number of L2 invalidations. Figure 2 shows the results (normalized 

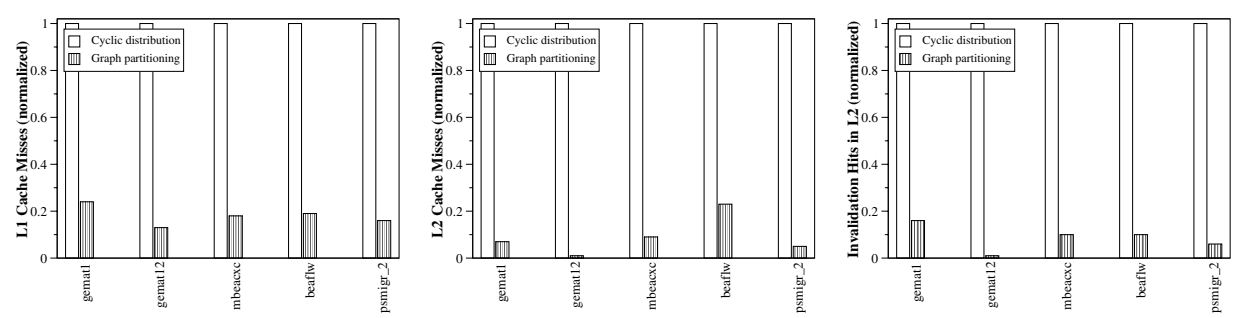

Fig. 2. Cache behavior

with respect to the cyclic distribution) for each test matrix on 8 processors. As can be observed, the reduction in the number of cache misses and invalidations is very significant.

Figure 3 shows the overall speedups (inspector and executor phases) on 8 processors for different workloads. Speedups were calculated with respect to the sequential execution of the code of Figure 1. Our proposal works better for loops with low $W$ because, in this case, memory hierarchy performance has a greater influence on the overall execution time. As $W$ increases, the improvement falls because load balancing and waiting times become critical factors for performance. The increase in the speedups illustrated in Figure 3 is a direct consequence of the improvement in data locality introduced by our approach. The best memory hierarchy optimization achieved by matrix gemat12 results in the highest increase in speedup.

In many applications, the loop to be parallelized is contained in one or more sequential loops. In this case, if the access pattern to array $A$ does not change across iterations, the inspector can be reused and thus its cost is amortized. An example of such applications are iterative sparse linear system solvers. Figure 4 shows the executor speedups on 8 processors for different workloads. Note that not only speedups increase, but also the improvement with respect to the cyclic iteration distribution strategy.
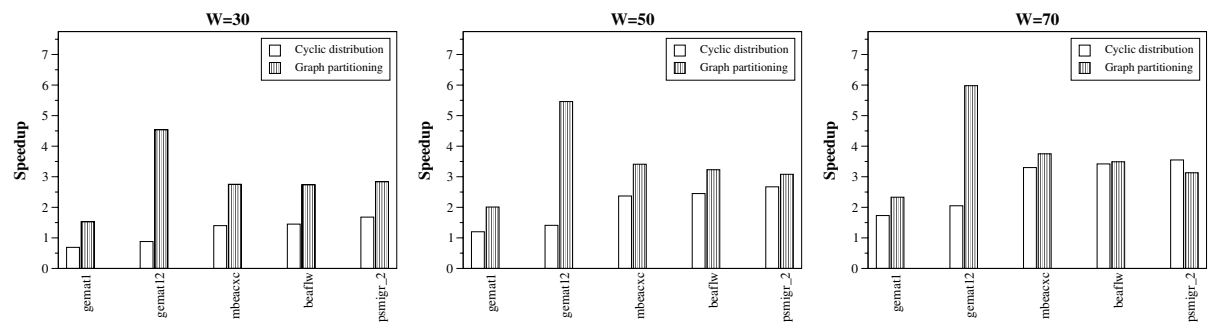

Fig. 3. Overall speedups on 8 processors for different workloads 

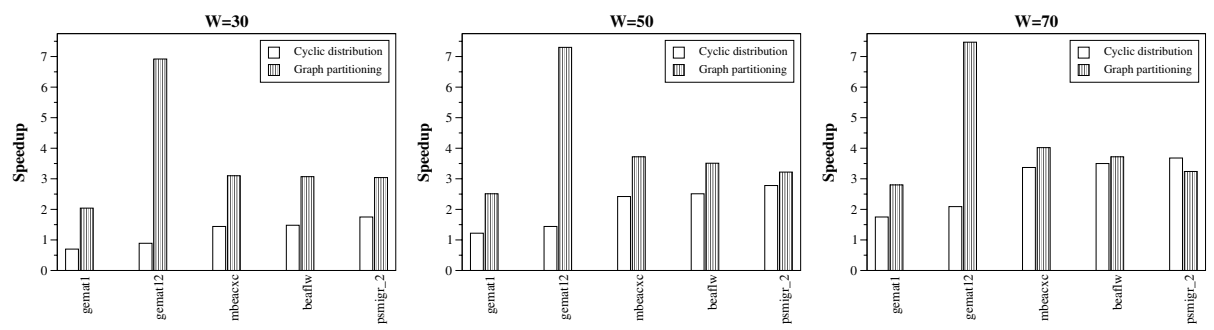

Fig. 4. Executor speedups on 8 processors for different workloads

\section{Conclusions}

Cache misses are becoming increasingly costly due to the widening gap between processor and memory performance. Therefore, it is a primary goal to increase the performance of each memory hierarchy level. In this work we have presented a proposal to parallelize doacross loops with indirect array accesses using run-time support. It is based on loop restructuring, and achieves important reductions in the number of cache misses and invalidations. It results in a significant increase in the achieved speedups (except for high workloads), and this improvement is even more significant if the inspector can be reused.

\section{References}

1. D.-K. Chen, J. Torrellas and P.-C. Yew: An Efficient Algorithm for the Run-Time Parallelization of DOACROSS Loops, Proc. Supercomputing Conf. (1994) 518-527

2. J.H. Saltz, R. Mirchandaney and K. Crowley: Run-Time Parallelization and Scheduling of Loops, IEEE Trans. on Computers 40(5) (1991) 603-612

3. C.-Z. Xu and V. Chaudhary: Time Stamp Algorithms for Runtime Parallelization of DOACROSS Loops with Dynamic Dependences, IEEE Trans. on Parallel and Distributed Systems 12(5) (2001) 433-450

4. C.-Q. Zhu and P.-C. Yew: A Scheme to Enforce Data Dependence on Large Multiprocessor Systems, IEEE Trans. on Soft. Eng. 13(6) (1987) 726-739

5. C. Xu: Effects of Parallelism Degree on Run-Time Parallelization of Loops, Proc. 31st Hawaii Int. Conf. on System Sciences (1998)

6. G. Karypis and V. Kumar: A Fast and High Quality Multilevel Scheme for Partitioning Irregular Graphs, SIAM J. on Scientific Comp. 20(1) (1999) 359-392

7. M.J. Martín, D.E. Singh, J. Touriño and F.F. Rivera: Exploiting Locality in the Run-time Parallelization of Irregular Loops, Proc. 2002 Int. Conf. on Parallel Processing (2002)

8. Y. Saad: SPARSKIT: a Basic Tool Kit for Sparse Matrix Computations (Version 2), at http://www.cs.umn.edu/Research/darpa/SPARSKIT/sparskit.html (1994)

9. I.S. Duff, R.G. Grimes and J.G.Lewis: User's Guide for the Harwell-Boeing Sparse Matrix Collection, Tech. Report TR-PA-92-96, CERFACS (1992) 